\title{
DIABETES IS REVERSIBLE - A REALITY
}

\section{Introduction}

537 million adults (20-79 years age group) approximately 1 in 11 are living with diabetes as per IDF 2021 data. This number is predicted to raise to 643 million by 2030 and 784 million by 2045. Over 4 in 5 (81\%) adults with diabetes live in low and middle income countries. ${ }^{1}$

Type 2 DM has long been identified as an incurable chronic disease based on traditional means of treatment. The best out come that has been expected is amelioration of diabetes symptoms, or slowing its inevitable progression. Approximately $50 \%$ of Type 2 DM patients will need insulin therapy within 10 years of diagnosis. ${ }^{2}$ Although in the past diabetes has been called chronic and irreversible, the paradigm is changing now. ${ }^{3,4}$ The recent 2016 WHO global report on diabetes added a section on diabetes reversal and acknowledged that it can be achieved through weight loss and calorie restricton. ${ }^{4}$

"Diabetes Reversal" is a term that best found its way into scientific articles and the lay press alike: "remission" has also been used. Criteria: While the exact criteria are still debated, most agree that a HbA1c $<6.5$ for an extended period of time without the use of glycemic control medications, except Metformin, as it has indications beyond diabetes. Likewise, terms such as "partial" (HbA1c <6.5) or "complete" $(\mathrm{HbA} 1 \mathrm{c}<5.7)$ without glycemic control medications for 1 year also used by an experts panel. It is important to note that the term "cure" has not been applied to type 2DM, as there does exists the potential for reoccurrence, which has been well documented in the literature.

Despite the growing evidence that reversal is possible, achieving reversal is not commonly encouraged by our health care system. In fact, reversal is not a goal in diabetes. Current standard of care does not lead to diabetes reversal. This raises the question of whether current standard of care is really the best practise. A large study by Kaiser et al., found diabetes remission rate of $0.3 \%$ with standard of care. ${ }^{6}$ The status quo approach will not reverse the health crisis of diabetes.

A significant number of studies indicate that diabetes reversal is achievable using bariatric surgery, while other approaches such as low-calorie diets (LCD) or carbohydrate restriction (LC), have also shown effectiveness in an increasing number of studies.

\section{Low-calorie diets (LCD)}

The number of studies examining diabetes reversal using non surgical techniques has increased. A few such studies have reported successful weight loss with decrease insulin resistance, plasma glucose, medications used following a LCD. As early as 1976, Bistrian et al., ${ }^{7}$ reported that a very low calorie protein sparing modified fast allowed for insulin elimination in all seven obese patient with type 2 DM. The average time to insulin discontinuation was only 6.5 days, the longest was 90 days. In a study by Bauman et al., a low calorie diet of $900 \mathrm{~K} \mathrm{Cal}$, including $150 \mathrm{~g}$ of protein, led to significant improvement in glycemic control. Furthermore a study conducted in obese type $2 \mathrm{DM}$ found that a LCD and gastric bypass surgery were equally effective in achieving weight loss and HbA1c control. ${ }^{8}$

A community based cluster-randomised clinical trial called DiRECT (diabetes remission clinical trial). DiRECT enrolled a sample of 306 relatively healthy participants with type $2 \mathrm{DM}$ (people on insulin or with a duration of diabetes longer than 6 years were excluded). They were cluster randomised to either standard diabetes care or an intervention using low calorie meal replacement diet (825 to $853 \mathrm{Kcal} /$ day) for 3 to 5 months, followed by step wise food reintroduction and long term weight maintenance program. At one year follow up $46 \%$ of patients went into diabetes reversal and $36 \%$ went into remission at the end of 2 years. $11 \%$ had lost greater than $15 \mathrm{~kg}$ weight. However in the standard care only 2 to $3 \%$ went into remission. Taken together evidence suggests that LCD is effective in reversing diabetes in the short term up to 2 years.

However it is crucial that further studies are directed towards assessing the long term sustainability of diabetes reversal by LCD and feasibility of this diet on the physiological adaptation and body composition changes.

\section{Carbohydrate-restricted Diets (LC)}

Before the discovery of insulin in 1921, low carbohydrate (CHO) diet were the only prescribed treatment for diabetes. The paradigm shifted both with the development of exogenous insulin and later with the emergence of low fat diet paradigm. A diet low in fat which by default is high in $\mathrm{CHO}$, became the standard recommendation in guidelines around the globe. Rather than preventing elevations in glucose, ${ }^{10}$ the goal became the maintenance of blood sugar control via the increased use of glycemic control medications including insulin. ${ }^{11}$ Over the last decade, clinical studies have begun to resurrect the pre- insulin LC dietary approach. LC diet has recently been endorsed as an eating pattern by the ADA and EASD. ${ }^{12,13}$ In addition the veterans affairs/ department of defence (VA/DOD) guidelines now recommend carbohydrate restrictions as low as $14 \%$ of energy in take. ${ }^{14}$

LC diets are based on macro nutrient changes rather than focus on calorie restriction. ${ }^{15}$ Although the exact definition varies a LC diet usually restricts total carbohydrates to less than $130 \mathrm{~g} /$ day, while a very LC or ketogenic diet usually restricts total carbohydrate to as low as 20 to 30 grams / day. Carbohydrate sources are primarily non starchy vegetables with some nuts, diary and limited fruits.

A total of 32 separate trials examining the $\mathrm{CHO}$ restriction as a treatment for type $2 \mathrm{DM}$ has been found in the literature $(16,17,18,19)$. A longer study 34 weeks (20) found that a very low $\mathrm{CHO}$ - ketogenic diet (20 to 50 grams $\mathrm{CHO}$ ) resulted in $\mathrm{HbA} 1 \mathrm{c}<6.5$ in $55 \%$ of patients compared to $0 \%$ in the low fat group. 
Although the use of very low $\mathrm{CHO}$ diets for diabetes reversal shows promising results the lack of long term follow up studies and also the adverse effects of high fat content of the diet and also a very low CHO diet is difficult to advice for Indian scenario who are by tradition has high carbohydrate intake.

\section{Green Mediterranean Diet}

New research suggests that higher levels of the hormone Ghrelin, which is produced from stomach during fasting or dieting , may help people lose fat(mainly visceral) and improve insulin sensitivity, helps in diabetes reversal. The green Mediterranean focuses on leafy green vegetables and excludes all red meat, and participants of such a diet had significant higher hormone Ghrelin levels. ${ }^{17}$

\section{Bariatric Surgery}

Bariatric Surgery has long been recognised as a potential treatment for both morbid obesity and the metabolic processes that accompany it , specifically type $2 \mathrm{DM}$. The effectiveness of diabetes reversal depends on the choice of surgical procedure, there is unilateral glycemic improvement following operation and surgery has been found to be superior to medical management. Accordingly, in 2016 the $2^{\text {nd }}$ diabetes surgery summit (DSS- II) released recommendations, endorsed by 45 medical and scientific societies worldwide, to use Bariatric Surgery as a treatment for type2 DM for adults with a BMI > 40 or $35>\mathrm{kg} / \mathrm{m} 2$ with obesity related co-morbidities.

Long term outcome of Bariatric Surgery depends on multiple factors, including type of surgery, patient co-morbidities, patient readiness for lifelong dietary changes and ongoing surveillance. while Surgery effectively reverses diabetes in upto $80 \%$ of the patients in the short term. Surgery effectiveness not only due to volume restriction and intestinal mal-absorption but also due to altered GI hormone release(decreased ghrelin, markedly increased GLP1, mildly increased GIP and CCK, PYY peptides) impacting the feeding behaviour via the gut - brain axis.

\section{Conclusions}

Over all as a society we can no longer afford or tolerate the continued raising rates of diabetes. Despite many barriers within the health care system as a whole, providers are responsible on a daily basis for the lives of patients caught up in this un precedented epidemic. The current standard of care may be suitable for some, but others would surely chose reversal if they understood "there was a choice". The choice can only be offered if providers are not only aware that "reversal is possible" but have the education needed to review these options in a patient-centric discussion.

Dr Mohan K Rao, MD

Fellowship in Diabetes \& Endocrinology

UTSW Medical Center (USA)

Endocrinologist RRMCH Diabetes Center

Medical Director \& Consultant Endocrinologist

NS Diabetes and Hormone Center

\section{References}

1. IDF Diabetes atlas.org

2. Home P., Riddle M et al., Insulin therapy in people with Type 2 DM. Opportunities and Challenges. Diabetes Care 2014;37:1499-1508

3. WHO report on diabetes; 2016 available online: https://www.who.int/diabetes/publications/grd-2016/en/

4. Davies MJ, Fradkin J et al., A consensus report by the ADA and EASD. Diabetes care 2018;41:2669-2701.

5. Ramos-Levi A.M., Cabrerizo et al., Which criteria to should be used to define type 2 diabetes remission after bariatric surgery. BMC Surgery 2013;13:8

6. Karter AJ, Nundy S. Et al., Incidence of remission in adults with Type 2 diabetes. The Diabetes care. 2014;37;3188-3195

7. Bistrian B.R., Blackburn et al., Nitrogen metabolism and insulin requirements in obese diabetic adults on a protein-sparing modified fast. Diabetes. 1976;25:494-504

8. Hughes TA, Gwyme et al., Effects of caloric restriction and weight loss on glycemic control. Am.J.Med. 1984;77:7-17

9. Mclnnes N, Smith A, Balasubramaniam K et al.,. Piloting a remission stratergy in type 2 diabetes. JCEM 2017;102:1596-1605

10. Arky R, Wylie- Rosett et al., Examination of current dietary recommendation in type 2 DM. Diabetes care 19825:59-63

11. Anderson JW, Geil P.B. et al., Am. J. Med. 1988;85:159-165

12. ADA smmary of Revisions: Standar of Medical Care in diabetes care. 2019;42:S4-S6

13. Department of veteran affairs and Department of Defence VA/DoD Clinical Practice guideline for the management of Type 2 DM in primary care. Online: https://www.healthquality.va.gov/guidelines/cd/diabetes

14. Stern L, Iqbal N et al., The effect of low carb diet Vs conventional weight loss diet. Ann. Intern. Med. 2004;140:778-785

15. Miyashita Y Ozaki H et al., Beneficial effect of low carb in low calorie diets on visceral fat reduction in type 2 diabetic patients with obesity. Diabetes Res. Clic. Pract. 2004;65:235-241

16. Saslow LR, Mason A.E, et al., low carb ketogenic diet in type 2 DM. A randomized controlled trial. J. Med. Int. Res. $2017 ; 19 ; \mathrm{e} 36$.

17. Gal Tsaban et al., Heart. 2020. 